Commun. Korean Math. Soc. 28 (2013), No. 1, pp. 87-105

http://dx.doi.org/10.4134/CKMS.2013.28.1.087

\title{
ON SOME GENERATING FUNCTIONS OF GENERALIZED BATEMAN'S AND PASTERNAK'S POLYNOMIALS OF TWO VARIABLES
}

\author{
Sayed Mohammad Abbas, Mumtaz Ahmad Khan, and Abdul Hakim Khan
}

\begin{abstract}
The aim of the present paper is to study some generating functions of generalized Bateman's and Pasternak's polynomials of two variables.
\end{abstract}

\section{Introduction}

In our earlier papers [1] and [2] we obtained some generating functions for Jacobi and generalized Rice polynomials of two variables respectively. In the present paper some generating functions for generalized Bateman's and Pasternak's polynomials of two variables have been obtained.

The following definitions are required in this paper:

The generalized Bateman's polynomial $Z_{n}^{(\alpha, \beta)}(b, x)$ is defined by Khan and Shukla [3]

$$
Z_{n}^{(\alpha, \beta)}(b, x)={ }_{2} F_{2}\left[\begin{array}{cc}
-n, 1+\alpha+\beta+n & ; \\
1+\alpha, 1+b & ;
\end{array}\right] .
$$

Another generalized Bateman's polynomial $F_{n}^{(\alpha, \beta)}(p, z)$ is defined by Khan and Shukla [3]

$$
F_{n}^{(\alpha, \beta)}(p, z)={ }_{3} F_{2}\left[\begin{array}{cc}
-n, 1+\alpha+\beta+n, & \frac{1}{2}(1+z) \\
1+\alpha, p & ; 1
\end{array}\right] .
$$

The generalized Pasternak's polynomial $F_{n, m}^{(\alpha, \beta)}(z)$ is defined by Khan and Shukla [3]

$$
F_{n, m}^{(\alpha, \beta)}(z)={ }_{3} F_{2}\left[\begin{array}{cc}
-n, 1+\alpha+\beta+n, \frac{1}{2}(1+z+m) & ; 1 \\
1+\alpha, 1+m & ;
\end{array}\right] .
$$

Received January 14, 2012; Revised March 11, 2012.

2010 Mathematics Subject Classification. 33C45.

Key words and phrases. Bateman's polynomial of one and two variables, Pasternak's polynomial of one and two variables, Kampé de Fériet series, triple hypergeometric series, generating functions. 
Kampé de Fériet series in the generalized form is defined by [6, p. 63, Eq. (16)]

$$
\begin{aligned}
& F_{l: m ; n}^{p: q ; k}\left[\begin{array}{lll}
\left(a_{p}\right):\left(b_{q}\right) & ;\left(c_{k}\right) ; \\
\left(\alpha_{l}\right):\left(\beta_{m}\right) & ;\left(\gamma_{n}\right) & ;
\end{array}\right] \\
& =\sum_{r=0}^{\infty} \sum_{s=0}^{\infty} \frac{\prod_{j=1}^{p}\left(a_{j}\right)_{r+s} \prod_{j=1}^{q}\left(b_{j}\right)_{r} \prod_{j=1}^{k}\left(c_{j}\right)_{s}}{\prod_{j=1}^{l}\left(\alpha_{j}\right)_{r+s} \prod_{j=1}^{m}\left(\beta_{j}\right)_{r} \prod_{j=1}^{n}\left(\gamma_{j}\right)_{s}} \frac{x^{r}}{r !} \frac{y^{s}}{s !} .
\end{aligned}
$$

The generalized hypergeometric functions of three variables $F^{(3)}[x, y, z]$ are defined as (see [5]):

$(1.5)$

$$
\begin{aligned}
& F^{(3)}\left[\begin{array}{r}
(a)::(b) ;\left(b^{\prime}\right) ;\left(b^{\prime \prime}\right):(c) ;\left(c^{\prime}\right) ;\left(c^{\prime \prime}\right) ; \\
(e)::(g) ;\left(g^{\prime}\right) ;\left(g^{\prime \prime}\right):(h) ;\left(h^{\prime}\right) ;\left(h^{\prime \prime}\right) ;
\end{array}, y, z\right] \\
= & \sum_{m=0}^{\infty} \sum_{n=0}^{\infty} \sum_{p=0}^{\infty} \frac{((a))_{m+n+p}((b))_{m+n}\left(\left(b^{\prime}\right)\right)_{n+p}\left(\left(b^{\prime \prime}\right)\right)_{m+p}((c))_{m}\left(\left(c^{\prime}\right)\right)_{n}\left(\left(c^{\prime \prime}\right)\right)_{p}}{((e))_{m+n+p}((g))_{m+n}\left(\left(g^{\prime}\right)\right)_{n+p}\left(\left(g^{\prime \prime}\right)\right)_{m+p}((h))_{m}\left(\left(h^{\prime}\right)\right)_{n}\left(\left(h^{\prime \prime}\right)\right)_{p}} \frac{x^{m}}{m !} \frac{y^{n}}{n !} \frac{z^{p}}{p !} .
\end{aligned}
$$

\section{Generalized Bateman's polynomials of two variables}

$$
Z_{n}^{\left(\alpha_{1}, \beta_{1} ; \alpha_{2}, \beta_{2}\right)}\left(b_{1}, x ; b_{2}, y\right)
$$

The generalized Bateman's polynomials of two variables $Z_{n}^{\left(\alpha_{1}, \beta_{1} ; \alpha_{2}, \beta_{2}\right)}\left(b_{1}, x\right.$; $\left.b_{2}, y\right)$ are defined by

$$
\begin{aligned}
& Z_{n}^{\left(\alpha_{1}, \beta_{1} ; \alpha_{2}, \beta_{2}\right)}\left(b_{1}, x ; b_{2}, y\right) \\
= & \sum_{r=0}^{n} \sum_{s=0}^{n-r} \frac{(-n)_{r+s}\left(1+\alpha_{1}+\beta_{1}+n\right)_{r}\left(1+\alpha_{2}+\beta_{2}+n\right)_{s}}{r ! s !\left(1+\alpha_{1}\right)_{r}\left(1+b_{1}\right)_{r}\left(1+\alpha_{2}\right)_{s}\left(1+b_{2}\right)_{s}} x^{r} y^{s} .
\end{aligned}
$$

The definition (2.1) can also be expressed in terms of double hypergeometric function as follows:

$$
\begin{aligned}
& Z_{n}^{\left(\alpha_{1}, \beta_{1} ; \alpha_{2}, \beta_{2}\right)}\left(b_{1}, x ; b_{2}, y\right) \\
= & F_{0: 2 ; 2}^{1: 1 ; 1}\left[\begin{array}{ccc}
-n & : 1+\alpha_{1}+\beta_{1}+n & ; 1+\alpha_{2}+\beta_{2}+n ; \\
- & : 1+\alpha_{1}, 1+b_{1} & ; 1+\alpha_{2}, 1+b_{2}
\end{array} ; x, y\right],
\end{aligned}
$$

where we have used a special case of the double hypergeometric function defined by (1.4).

The definition (2.1) can also be represented as follows:

$$
\begin{aligned}
& Z_{n}^{\left(\alpha_{1}, \beta_{1} ; \alpha_{2}, \beta_{2}\right)}\left(b_{1}, x ; b_{2}, y\right) \\
= & \sum_{r=0}^{n} \frac{(-n)_{r}\left(1+\alpha_{1}+\beta_{1}+n\right)_{r} x^{r}}{r !\left(1+\alpha_{1}\right)_{r}\left(1+b_{1}\right)_{r}} Z_{n-r}^{\left(\alpha_{2}, \beta_{2}+r\right)}\left(b_{2}, y\right),
\end{aligned}
$$

where $Z_{n}^{(\alpha, \beta)}\left(b_{2}, y\right)$ is the well known generalized Bateman's polynomial of one variable defined by (1.1). 
The relationships between generalized Bateman's polynomials of two variables and generalized Bateman's polynomials of single variable are as follows:

$$
Z_{n}^{\left(\alpha_{1}, \beta_{1} ; \alpha_{2}, \beta_{2}\right)}\left(b_{1}, x ; b_{2}, 0\right)=Z_{n}^{\left(\alpha_{1}, \beta_{1}\right)}\left(b_{1}, x\right)
$$

and

$$
Z_{n}^{\left(\alpha_{1}, \beta_{1} ; \alpha_{2}, \beta_{2}\right)}\left(b_{1}, 0 ; b_{2}, y\right)=Z_{n}^{\left(\alpha_{2}, \beta_{2}\right)}\left(b_{2}, y\right)
$$

\section{Generating functions for generalized Bateman's polynomials}

$$
Z_{n}^{\left(\alpha_{1}, \beta_{1} ; \alpha_{2}, \beta_{2}\right)}\left(b_{1}, x ; b_{2}, y\right)
$$

The polynomials $Z_{n}^{\left(\alpha_{1}, \beta_{1} ; \alpha_{2}, \beta_{2}\right)}\left(b_{1}, x ; b_{2}, y\right)$ admit the following generating functions:

$$
\begin{aligned}
& \sum_{n=0}^{\infty} \frac{(\lambda)_{n}}{n !} Z_{n}^{\left(\alpha_{1}, \beta_{1} ; \alpha_{2}, \beta_{2}\right)}\left(b_{1}, x ; b_{2}, y\right) t^{n} \\
& =F_{2: 0 ; 2 ; 2}^{3: 0 ; 0 ; 0}\left[\begin{array}{ll}
(\lambda: 1,1,1),\left(1+\alpha_{1}+\beta_{1}: 1,2,1\right),\left(1+\alpha_{2}+\beta_{2}: 1,1,2\right) & :-; \\
\left(1+\alpha_{1}+\beta_{1}: 1,1,1\right),\left(1+\alpha_{2}+\beta_{2}: 1,1,1\right) & :-;
\end{array}\right. \\
& \left.\left(1+\alpha_{1}: \overline{1}\right),\left(1+b_{1}: 1\right) \stackrel{;}{;}\left(1+\alpha_{2}: \overline{1}\right),\left(1+b_{2}: 1\right) \stackrel{;}{;},-x t,-y t\right],
\end{aligned}
$$

where we have used a special case of generalized Lauricella function (see, for example, $[5$, p. 37]).

$$
\begin{aligned}
& \sum_{n=0}^{\infty} \frac{1}{n !(\mu)_{n}} Z_{n}^{\left(\alpha_{1}, \beta_{1} ; \alpha_{2}, \beta_{2}\right)}\left(b_{1}, x ; b_{2}, y\right) t^{n} \\
& =F_{3: 0 ; 2 ; 2}^{2: 0 ; 0 ; 0}\left[\begin{array}{ll}
\left(1+\alpha_{1}+\beta_{1}: 1,2,1\right),\left(1+\alpha_{2}+\beta_{2}: 1,1,2\right) & :-; \\
(\mu: 1,1,1),\left(1+\alpha_{1}+\beta_{1}: 1,1,1\right),\left(1+\alpha_{2}+\beta_{2}: 1,1,1\right) & :-;
\end{array}\right. \\
& \left.\left(1+\alpha_{1}: \overline{1}\right),\left(1+b_{1}: 1\right) \stackrel{;}{;}\left(1+\alpha_{2}: \overline{1}\right),\left(1+b_{2}: 1\right) ; t,-x t,-y t\right] .
\end{aligned}
$$

$$
\begin{aligned}
& \sum_{n=0}^{\infty} \frac{(\lambda)_{n}}{n !(\mu)_{n}} Z_{n}^{\left(\alpha_{1}, \beta_{1} ; \alpha_{2}, \beta_{2}\right)}\left(b_{1}, x ; b_{2}, y\right) t^{n} \\
& =F_{3: 0 ; 2 ; 2}^{3: 0 ; 0 ; 0}\left[\begin{array}{l}
(\lambda: 1,1,1),\left(1+\alpha_{1}+\beta_{1}: 1,2,1\right),\left(1+\alpha_{2}+\beta_{2}: 1,1,2\right):-; \\
(\mu: 1,1,1),\left(1+\alpha_{1}+\beta_{1}: 1,1,1\right),\left(1+\alpha_{2}+\beta_{2}: 1,1,1\right):-;
\end{array}\right. \\
& \left.\left(1+\alpha_{1}: \overline{1}\right),\left(1+b_{1}: 1\right) \stackrel{;}{;}\left(1+\alpha_{2}: \overline{1}\right),\left(1+b_{2}: 1\right) \stackrel{;}{;}, t,-x t,-y t\right] .
\end{aligned}
$$


(3.4)

$$
\begin{aligned}
& \sum_{n=0}^{\infty} \frac{\left(\lambda_{1}\right)_{n}\left(\lambda_{2}\right)_{n}}{n !(\mu)_{n}} Z_{n}^{\left(\alpha_{1}, \beta_{1} ; \alpha_{2}, \beta_{2}\right)}\left(b_{1}, x ; b_{2}, y\right) t^{n} \\
&=F_{3: 0 ; 2 ; 2}^{4: 0 ; 0 ; 0}\left[\begin{array}{c}
\left(\lambda_{1}: 1,1,1\right),\left(\lambda_{2}: 1,1,1\right),\left(1+\alpha_{1}+\beta_{1}: 1,2,1\right),\left(1+\alpha_{2}+\beta_{2}: 1,1,2\right) \\
(\mu: 1,1,1),\left(1+\alpha_{1}+\beta_{1}: 1,1,1\right),\left(1+\alpha_{2}+\beta_{2}: 1,1,1\right)
\end{array}\right. \\
& \quad-; \quad \begin{array}{c}
; \\
\quad
\end{array} \\
& \quad ;\left(1+\alpha_{1}: 1\right),\left(1+b_{1}: 1\right) ;\left(1+\alpha_{2}: 1\right),\left(1+b_{2}: 1\right) ;
\end{aligned}
$$

$$
\begin{aligned}
& \sum_{n=0}^{\infty} \frac{\left(\lambda_{1}\right)_{n}\left(\lambda_{2}\right)_{n}}{n !\left(\mu_{1}\right)_{n}\left(\mu_{2}\right)_{n}} Z_{n}^{\left(\alpha_{1}, \beta_{1} ; \alpha_{2}, \beta_{2}\right)}\left(b_{1}, x ; b_{2}, y\right) t^{n} \\
& =F_{4: 0 ; 2 ; 2}^{4: 0 ; 0 ;}\left[\begin{array}{l}
\left(\lambda_{1}: 1,1,1\right),\left(\lambda_{2}: 1,1,1\right),\left(1+\alpha_{1}+\beta_{1}: 1,2,1\right),\left(1+\alpha_{2}+\beta_{2}: 1,1,2\right): \\
\left(\mu_{1}: 1,1,1\right),\left(\mu_{2}: 1,1,1\right),\left(1+\alpha_{1}+\beta_{1}: 1,1,1\right),\left(1+\alpha_{2}+\beta_{2}: 1,1,1\right):
\end{array}\right. \\
& \text { - } \left.\left.\quad ; \quad-\overline{\alpha_{1}}: 1\right),\left(1+b_{1}: 1\right) ;\left(1+\alpha_{2}: 1\right),\left(1+b_{2}: 1\right) ; t,-x t,-y t\right] .
\end{aligned}
$$

\section{Special cases}

If we put $\lambda=\mu$ in the generating function (3.3), we get (3.6)

$$
\begin{aligned}
& \sum_{n=0}^{\infty} \frac{1}{n !} Z_{n}^{\left(\alpha_{1}, \beta_{1} ; \alpha_{2}, \beta_{2}\right)}\left(b_{1}, x ; b_{2}, y\right) t^{n} \\
& =F_{2: 0 ; 2 ; 2}^{2: 0 ; 0 ; 0}\left[\begin{array}{l}
\left(1+\alpha_{1}+\beta_{1}: 1,2,1\right),\left(1+\alpha_{2}+\beta_{2}: 1,1,2\right):-; \\
\left(1+\alpha_{1}+\beta_{1}: 1,1,1\right),\left(1+\alpha_{2}+\beta_{2}: 1,1,1\right):-;
\end{array}\right. \\
& \left.\left(1+\alpha_{1}: \overline{1}\right),\left(1+b_{1}: 1\right) ;\left(1+\alpha_{2}: \overline{1}\right),\left(1+b_{2}: 1\right) ; t,-x t,-y t\right] .
\end{aligned}
$$

When $\lambda_{1}=1+\alpha_{1}+\beta_{1}$ and $\lambda_{2}=1+\alpha_{2}+\beta_{2}$, then the generating function (3.4) reduces immediately to the form:

$$
\begin{aligned}
& \sum_{n=0}^{\infty} \frac{\left(1+\alpha_{1}+\beta_{1}\right)_{n}\left(1+\alpha_{2}+\beta_{2}\right)_{n}}{n !(\mu)_{n}} Z_{n}^{\left(\alpha_{1}, \beta_{1} ; \alpha_{2}, \beta_{2}\right)}\left(b_{1}, x ; b_{2}, y\right) t^{n} \\
& =F_{1: 0 ; 2 ; 2}^{2: 0 ; 0 ; 0}\left[\begin{array}{cl}
\left(1+\alpha_{1}+\beta_{1}: 1,2,1\right),\left(1+\alpha_{2}+\beta_{2}: 1,1,2\right) & :-; \\
(\mu: 1,1,1) & :-;
\end{array}\right. \\
& \left.\left(1+\alpha_{1}: \overline{1}\right),\left(1+b_{1}: 1\right) \stackrel{;}{;}\left(1+\alpha_{2}: \overline{1}\right),\left(1+b_{2}: 1\right) \stackrel{;}{;} t,-x t,-y t\right] .
\end{aligned}
$$


Further, the sequence $\left\{Z_{n}^{\left(\alpha_{1}, \beta_{1} ; \alpha_{2}, \beta_{2}\right)}\left(b_{1}, x ; b_{2}, y\right)\right\}_{n \in \mathbb{N}}$ admits the following generating functions:

$$
\begin{aligned}
& \sum_{n=0}^{\infty} \frac{1}{n !} Z_{n}^{\left(\alpha_{1}, \beta_{1}-n ; \alpha_{2}, \beta_{2}-n\right)}\left(b_{1}, x ; b_{2}, y\right) t^{n} \\
= & e^{t}{ }_{1} F_{2}\left[\begin{array}{c}
1+\alpha_{1}+\beta_{1} ; \\
1+\alpha_{1}, 1+b_{1} ;
\end{array}-x t\right]{ }_{1} F_{2}\left[\begin{array}{c}
1+\alpha_{2}+\beta_{2} ; \\
1+\alpha_{2}, 1+b_{2} ;
\end{array} ;-y t\right] ;
\end{aligned}
$$

$$
\begin{aligned}
& \sum_{n=0}^{\infty} \frac{\left(-\alpha_{1}\right)_{n}}{n !} Z_{n}^{\left(\alpha_{1}-n, \beta_{1} ; \alpha_{2}, \beta_{2}-n\right)}\left(b_{1}, x ; b_{2}, y\right) t^{n} \\
= & (1-t)^{\alpha_{1}}{ }_{1} F_{1}\left[\begin{array}{cc}
1+\alpha_{1}+\beta_{1} \\
1+b_{1}
\end{array} ; x t\right]{ }_{2} F_{2}\left[\begin{array}{cc}
-\alpha_{1}, 1+\alpha_{2}+\beta_{2} & ; \\
1+\alpha_{2}, 1+b_{2} & ;
\end{array}\right]
\end{aligned}
$$

$$
\sum_{n=0}^{\infty} \frac{\left(-\alpha_{2}\right)_{n}}{n !} Z_{n}^{\left(\alpha_{1}, \beta_{1}-n ; \alpha_{2}-n, \beta_{2}\right)}\left(b_{1}, x ; b_{2}, y\right) t^{n}
$$

$=(1-t)^{\alpha_{2}}{ }_{1} F_{1}\left[\begin{array}{cc}1+\alpha_{2}+\beta_{2} & ; \\ 1+b_{2} & ;\end{array} y t\right]{ }_{2} F_{2}\left[\begin{array}{cc}-\alpha_{2}, 1+\alpha_{1}+\beta_{1} & ; \\ 1+\alpha_{1}, 1+b_{1} & ;\end{array}\right]$

$$
\sum_{n=0}^{\infty} \frac{\left(-b_{1}\right)_{n}}{n !} Z_{n}^{\left(\alpha_{1}, \beta_{1}-n ; \alpha_{2}, \beta_{2}-n\right)}\left(b_{1}-n, x ; b_{2}, y\right) t^{n}
$$

$=(1-t)^{b_{1}}{ }_{1} F_{1}\left[\begin{array}{cc}1+\alpha_{1}+\beta_{1} & ; \\ 1+\alpha_{1} & ;\end{array}\right]$ t ${ }_{2} F_{2}\left[\begin{array}{c}1+\alpha_{2}+\beta_{2},-b_{1} \\ 1+\alpha_{2}, 1+b_{2}\end{array} ; \begin{array}{c}-y t \\ 1-t\end{array}\right]$;

$$
\sum_{n=0}^{\infty} \frac{\left(-b_{2}\right)_{n}}{n !} Z_{n}^{\left(\alpha_{1}, \beta_{1}-n ; \alpha_{2}, \beta_{2}-n\right)}\left(b_{1}, x ; b_{2}-n, y\right) t^{n}
$$

$=(1-t)^{b_{2}}{ }_{1} F_{1}\left[\begin{array}{cc}1+\alpha_{2}+\beta_{2} & ; \\ 1+\alpha_{2} & ;\end{array} y t\right]{ }_{2} F_{2}\left[\begin{array}{cc}1+\alpha_{1}+\beta_{1},-b_{2} & ; \\ 1+\alpha_{1}, 1+b_{1} & ;\end{array}\right]$;-t

$$
\sum_{n=0}^{\infty} \frac{(\lambda)_{n}}{n !} Z_{n}^{\left(\alpha_{1}, \beta_{1}-n ; \alpha_{2}, \beta_{2}-n\right)}\left(b_{1}, x ; b_{2}, y\right) t^{n}
$$

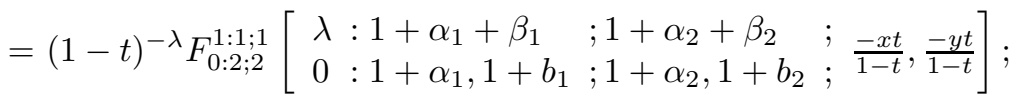

$$
\begin{aligned}
& \sum_{n=0}^{\infty} \frac{\left(-\alpha_{1}\right)_{n}}{n !\left(-\alpha_{1}-\beta_{1}\right)_{n}} Z_{n}^{\left(\alpha_{1}-n, \beta_{1}-n ; \alpha_{2}, \beta_{2}-n\right)}\left(b_{1}, x ; b_{2}, y\right) t^{n} \\
= & { }_{0} F_{1}\left[\begin{array}{cc}
- & ; \\
1+b_{1} ; & -x t
\end{array}\right] F_{1: 0 ; 2}^{1: 0 ; 1}\left[\begin{array}{cc}
-\alpha_{1} & :-; 1+\alpha_{2}+\beta_{2} \\
-\alpha_{1}-\beta_{1}:-; 1+\alpha_{2}, 1+b_{2} & ;
\end{array} \text { t, } t, y t\right] ;
\end{aligned}
$$


(3.15)

$$
\begin{aligned}
& \sum_{n=0}^{\infty} \frac{\left(-\alpha_{2}\right)_{n}}{n !\left(-\alpha_{2}-\beta_{2}\right)_{n}} Z_{n}^{\left(\alpha_{1}, \beta_{1}-n ; \alpha_{2}-n, \beta_{2}-n\right)}\left(b_{1}, x ; b_{2}, y\right) t^{n} \\
= & { }_{0} F_{1}\left[\begin{array}{cc}
- & ; \\
1+b_{2} ; & -y t
\end{array}\right] F_{1: 0 ; 2}^{1: 0 ; 1}\left[\begin{array}{cr}
-\alpha_{2} & :-; 1+\alpha_{1}+\beta_{1} \\
-\alpha_{2}-\beta_{2} & :-; 1+\alpha_{1}, 1+b_{1} ;
\end{array} ;,-x t\right] ;
\end{aligned}
$$

$$
\begin{aligned}
& \sum_{n=0}^{\infty} \frac{\left(-b_{1}\right)_{n}}{n !\left(-\alpha_{1}-\beta_{1}\right)_{n}} Z_{n}^{\left(\alpha_{1}, \beta_{1}-2 n ; \alpha_{2}, \beta_{2}-n\right)}\left(b_{1}-n, x ; b_{2}, y\right) t^{n} \\
= & { }_{0} F_{1}\left[\begin{array}{cc}
- & ; \\
1+\alpha_{1} ; & -x t
\end{array}\right] F_{1: 0 ; 2}^{1: 0 ; 1}\left[\begin{array}{cc}
-b_{1} & :-; 1+\alpha_{2}+\beta_{2} \\
-\alpha_{1}-\beta_{1}:-; 1+\alpha_{2}, 1+b_{2} & ;
\end{array} \text {; } t,-y t\right] ;
\end{aligned}
$$

$$
\begin{aligned}
& \sum_{n=0}^{\infty} \frac{\left(-b_{2}\right)_{n}}{n !\left(-\alpha_{2}-\beta_{2}\right)_{n}} Z_{n}^{\left(\alpha_{1}, \beta_{1}-n ; \alpha_{2}, \beta_{2}-2 n\right)}\left(b_{1}, x ; b_{2}-n, y\right) t^{n} \\
= & { }_{0} F_{1}\left[\begin{array}{cc}
- & ; \\
1+\alpha_{2} ; & -y t
\end{array}\right] F_{1: 0 ; 2}^{1: 0 ; 1}\left[\begin{array}{cc}
-b_{2} & :-; 1+\alpha_{1}+\beta_{1} \\
-\alpha_{2}-\beta_{2} & :-; 1+\alpha_{1}, 1+b_{1}
\end{array} ; t,-x t\right] .
\end{aligned}
$$

Also, we have the following generating functions:

$$
\begin{aligned}
& \sum_{n=0}^{\infty} \frac{(\lambda)_{n}}{n !(\mu)_{n}} Z_{n}^{\left(\alpha_{1}, \beta_{1}-n ; \alpha_{2}, \beta_{2}-n\right)}\left(b_{1}, x ; b_{2}, y\right) t^{n} \\
= & F^{(3)}\left[\begin{array}{lll}
\lambda::-;-;-:-; 1+\alpha_{1}+\beta_{1} & ; 1+\alpha_{2}+\beta_{2} \\
\mu::-;-;-:-; 1+\alpha_{1}, 1+b_{1} & ; 1+\alpha_{2}, 1+b_{2}
\end{array} ; t,-x t,-y t\right] ;
\end{aligned}
$$

$$
\begin{aligned}
& \sum_{n=0}^{\infty} \frac{\left(-\alpha_{1}\right)_{n}\left(-\alpha_{2}\right)_{n}}{n !} Z_{n}^{\left(\alpha_{1}-n, \beta_{1} ; \alpha_{2}-n, \beta_{2}\right)}\left(b_{1}, x ; b_{2}, y\right) t^{n} \\
= & \left.F^{(3)}\left[\begin{array}{llll}
-::-\alpha_{2} & ;-;-\alpha_{1}:-; 1+\alpha_{1}+\beta_{1} & ; 1+\alpha_{2}+\beta_{2} & ; \\
-::- & ;-;- & :-; 1+b_{1} & ; 1+b_{2}
\end{array}\right] \text { t } x t, y t\right] ;
\end{aligned}
$$

$$
\begin{aligned}
& \sum_{n=0}^{\infty} \frac{\left(-b_{1}\right)_{n}\left(-b_{2}\right)_{n}}{n !} Z_{n}^{\left(\alpha_{1}, \beta_{1}-n ; \alpha_{2}, \beta_{2}-n\right)}\left(b_{1}-n, x ; b_{2}-n, y\right) t^{n} \\
= & F^{(3)}\left[\begin{array}{llll}
-::-b_{2} & ;-;-b_{1}:-; 1+\alpha_{1}+\beta_{1} & ; 1+\alpha_{2}+\beta_{2} & ; \\
-::- & ;-;- & :-; 1+\alpha_{1} & ; 1+\alpha_{2}
\end{array} \text { t, xt, yt }\right]
\end{aligned}
$$

$$
\begin{aligned}
& \sum_{n=0}^{\infty} \frac{\left(-\alpha_{1}\right)_{n}\left(-\alpha_{2}\right)_{n}}{n !\left(-\alpha_{1}-\beta_{1}\right)_{n}\left(-\alpha_{2}-\beta_{2}\right)_{n}} Z_{n}^{\left(\alpha_{1}-n, \beta_{1}-n ; \alpha_{2}-n, \beta_{2}-n\right)}\left(b_{1}, x ; b_{2}, y\right) t^{n}
\end{aligned}
$$

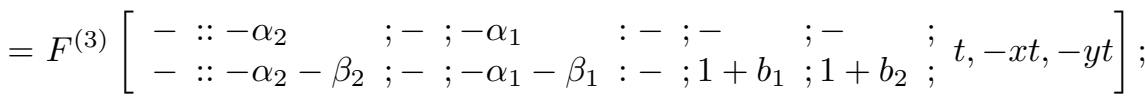




$$
\begin{aligned}
& \sum_{n=0}^{\infty} \frac{\left(-b_{1}\right)_{n}\left(-b_{2}\right)_{n}}{n !\left(-\alpha_{1}-\beta_{1}\right)_{n}\left(-\alpha_{2}-\beta_{2}\right)_{n}} Z_{n}^{\left(\alpha_{1}, \beta_{1}-2 n ; \alpha_{2}, \beta_{2}-2 n\right)}\left(b_{1}-n, x ; b_{2}-n, y\right) t^{n} \\
& =F^{(3)}\left[\begin{array}{llll}
-::-b_{2} & ;-;-b_{1} & :-;- & ;-
\end{array} \quad ; t,-x t,-y t\right] .
\end{aligned}
$$

\section{Generalized Bateman's polynomials of two variables $F_{n}^{\left(\alpha_{1}, \beta_{1} ; \alpha_{2}, \beta_{2}\right)}\left(p_{1}, z_{1} ; p_{2}, z_{2}\right)$}

Another generalized Bateman's polynomials of two variables $F_{n}^{\left(\alpha_{1}, \beta_{1} ; \alpha_{2}, \beta_{2}\right)}$ $\left(p_{1}, z_{1} ; p_{2}, z_{2}\right)$ are defined by

$$
\begin{aligned}
& F_{n}^{\left(\alpha_{1}, \beta_{1} ; \alpha_{2}, \beta_{2}\right)}\left(p_{1}, z_{1} ; p_{2}, z_{2}\right) \\
= & \sum_{r=0}^{n} \sum_{s=0}^{n-r} \frac{(-n)_{r+s}\left(1+\alpha_{1}+\beta_{1}+n\right)_{r}\left(\frac{1}{2}\left(1+z_{1}\right)\right)_{r}\left(1+\alpha_{2}+\beta_{2}+n\right)_{s}\left(\frac{1}{2}\left(1+z_{2}\right)\right)_{s}}{r ! s !\left(1+\alpha_{1}\right)_{r}\left(p_{1}\right)_{r}\left(1+\alpha_{2}\right)_{s}\left(p_{2}\right)_{s}} .
\end{aligned}
$$

The relation (4.1) can also be expressed in terms of double hypergeometric function as follows:

$$
\begin{aligned}
& F_{n}^{\left(\alpha_{1}, \beta_{1} ; \alpha_{2}, \beta_{2}\right)}\left(p_{1}, z_{1} ; p_{2}, z_{2}\right) \\
= & F_{0: 2 ; 2}^{1: 2 ; 2}\left[\begin{array}{lll}
-n: 1+\alpha_{1}+\beta_{1}+n, \frac{1}{2}\left(1+z_{1}\right) & ; 1+\alpha_{2}+\beta_{2}+n, \frac{1}{2}\left(1+z_{2}\right) ; 1,1 \\
-: 1+\alpha_{1}, p_{1} & ; 1+\alpha_{2}, p_{2} & ;
\end{array}\right],
\end{aligned}
$$

where we have used a special case of the double hypergeometric function defined by (1.4).

The definition (4.1) can also be represented as follows:

$$
\begin{aligned}
& F_{n}^{\left(\alpha_{1}, \beta_{1} ; \alpha_{2}, \beta_{2}\right)}\left(p_{1}, z_{1} ; p_{2}, z_{2}\right) \\
= & \sum_{r=0}^{n} \frac{(-n)_{r}\left(1+\alpha_{1}+\beta_{1}+n\right)_{r}\left(\frac{1}{2}\left(1+z_{1}\right)\right)_{r}}{r !\left(1+\alpha_{1}\right)_{r}\left(p_{1}\right)_{r}} F_{n-r}^{\left(\alpha_{2}, \beta_{2}+r\right)}\left(p_{2}, z_{2}\right),
\end{aligned}
$$

where $F_{n}^{(\alpha, \beta)}\left(p_{2}, z\right)$ is the well known generalized Bateman's polynomial of one variable defined by (1.2).

The relationships between generalized Bateman's polynomials of two variables and generalized Bateman's polynomials of single variable are as follows:

$$
F_{n}^{\left(\alpha_{1}, \beta_{1} ; \alpha_{2}, \beta_{2}\right)}\left(p_{1}, z_{1} ; p_{2},-1\right)=F_{n}^{\left(\alpha_{1}, \beta_{1}\right)}\left(p_{1}, z_{1}\right)
$$

and

$$
F_{n}^{\left(\alpha_{1}, \beta_{1} ; \alpha_{2}, \beta_{2}\right)}\left(p_{1},-1 ; p_{2}, z_{2}\right)=F_{n}^{\left(\alpha_{2}, \beta_{2}\right)}\left(p_{2}, z_{2}\right)
$$




\section{Generating functions for generalized Bateman's polynomials}

$$
F_{n}^{\left(\alpha_{1}, \beta_{1} ; \alpha_{2}, \beta_{2}\right)}\left(p_{1}, z_{1} ; p_{2}, z_{2}\right)
$$

The polynomials $F_{n}^{\left(\alpha_{1}, \beta_{1} ; \alpha_{2}, \beta_{2}\right)}\left(p_{1}, z_{1} ; p_{2}, z_{2}\right)$ admit the following generating functions:

(5.1)

$$
\begin{aligned}
& \sum_{n=0}^{\infty} \frac{(\lambda)_{n}}{n !} F_{n}^{\left(\alpha_{1}, \beta_{1} ; \alpha_{2}, \beta_{2}\right)}\left(p_{1}, z_{1} ; p_{2}, z_{2}\right) t^{n} \\
&=F_{2: 0 ; 2 ; 2}^{3: 0 ; 1 ; 1}\left[\begin{array}{ll}
(\lambda: 1,1,1),\left(1+\alpha_{1}+\beta_{1}: 1,2,1\right),\left(1+\alpha_{2}+\beta_{2}: 1,1,2\right):-; \\
\left(1+\alpha_{1}+\beta_{1}: 1,1,1\right),\left(1+\alpha_{2}+\beta_{2}: 1,1,1\right)
\end{array}\right. \\
&\left.\quad\left(\frac{1}{2}\left(1+z_{1}\right): 1\right) \quad ; \quad ;\left(\frac{1}{2}\left(1+z_{2}\right): 1\right) \quad ; t,-t,-t\right] \\
& \quad\left(1+\alpha_{1}: 1\right),\left(p_{1}: 1\right) ;\left(1+\alpha_{2}: 1\right),\left(p_{2}: 1\right) ;
\end{aligned}
$$

where we have used a special case of generalized Lauricella function (see, for example, [5, p. 37].

$(5.2)$

$$
\begin{aligned}
& \sum_{n=0}^{\infty} \frac{1}{n !(\mu)_{n}} F_{n}^{\left(\alpha_{1}, \beta_{1} ; \alpha_{2}, \beta_{2}\right)}\left(p_{1}, z_{1} ; p_{2}, z_{2}\right) t^{n}:-; \\
&=F_{3: 0 ; 2 ; 2}^{2: 0 ; 1 ; 1}\left[\begin{array}{rr}
\left(1+\alpha_{1}+\beta_{1}: 1,2,1\right),\left(1+\alpha_{2}+\beta_{2}: 1,1,2\right) \\
(\mu: 1,1,1),\left(1+\alpha_{1}+\beta_{1}: 1,1,1\right),\left(1+\alpha_{2}+\beta_{2}: 1,1,1\right):-;
\end{array}\right. \\
&\left(\begin{array}{ll}
\left.\frac{1}{2}\left(1+z_{1}\right): 1\right) & \left.;\left(\frac{1}{2}\left(1+z_{2}\right): 1\right) \quad ; t,-t,-t\right] \\
& \left(1+\alpha_{1}: 1\right),\left(p_{1}: 1\right) ;\left(1+\alpha_{2}: 1\right),\left(p_{2}: 1\right) ;
\end{array}\right.
\end{aligned}
$$

$$
\begin{aligned}
& \sum_{n=0}^{\infty} \frac{(\lambda)_{n}}{n !(\mu)_{n}} F_{n}^{\left(\alpha_{1}, \beta_{1} ; \alpha_{2}, \beta_{2}\right)}\left(p_{1}, z_{1} ; p_{2}, z_{2}\right) t^{n} \\
& =F_{3: 0 ; 2 ; 2}^{3: 0 ; 1 ; 1}\left[\begin{array}{l}
(\lambda: 1,1,1),\left(1+\alpha_{1}+\beta_{1}: 1,2,1\right),\left(1+\alpha_{2}+\beta_{2}: 1,1,2\right):-; \\
(\mu: 1,1,1),\left(1+\alpha_{1}+\beta_{1}: 1,1,1\right),\left(1+\alpha_{2}+\beta_{2}: 1,1,1\right):-;
\end{array}\right. \\
& \left.\begin{array}{lll}
\left(\frac{1}{2}\left(1+z_{1}\right): 1\right) & ;\left(\frac{1}{2}\left(1+z_{2}\right): 1\right) & ; \\
\left(1+\alpha_{1}: 1\right),\left(p_{1}: 1\right) & ;\left(1+\alpha_{2}: 1\right),\left(p_{2}: 1\right)
\end{array} ;,-t,-t\right] ;
\end{aligned}
$$

$$
\begin{aligned}
& \sum_{n=0}^{\infty} \frac{\left(\lambda_{1}\right)_{n}\left(\lambda_{2}\right)_{n}}{n !(\mu)_{n}} F_{n}^{\left(\alpha_{1}, \beta_{1} ; \alpha_{2}, \beta_{2}\right)}\left(p_{1}, z_{1} ; p_{2}, z_{2}\right) t^{n} \\
& =F_{3: 0 ; 2 ; 2}^{4: 0 ; 1 ; 1}\left[\begin{array}{c}
\left(\lambda_{1}: 1,1,1\right),\left(\lambda_{2}: 1,1,1\right),\left(1+\alpha_{1}+\beta_{1}: 1,2,1\right),\left(1+\alpha_{2}+\beta_{2}: 1,1,2\right): \\
(\mu: 1,1,1),\left(1+\alpha_{1}+\beta_{1}: 1,1,1\right),\left(1+\alpha_{2}+\beta_{2}: 1,1,1\right)
\end{array}:\right. \\
& \left.\begin{array}{lll}
-;\left(\frac{1}{2}\left(1+z_{1}\right): 1\right) & ;\left(\frac{1}{2}\left(1+z_{2}\right): 1\right) & ; \\
-;\left(1+\alpha_{1}: 1\right),\left(p_{1}: 1\right) & ;\left(1+\alpha_{2}: 1\right),\left(p_{2}: 1\right) & ;
\end{array},-t,-t\right] ;
\end{aligned}
$$


(5.5)

$$
\begin{aligned}
& \sum_{n=0}^{\infty} \frac{\left(\lambda_{1}\right)_{n}\left(\lambda_{2}\right)_{n}}{n !\left(\mu_{1}\right)_{n}\left(\mu_{2}\right)_{n}} F_{n}^{\left(\alpha_{1}, \beta_{1} ; \alpha_{2}, \beta_{2}\right)}\left(p_{1}, z_{1} ; p_{2}, z_{2}\right) t^{n} \\
& =F_{4: 0 ; 2 ; 2}^{4: 0 ; 1 ; 1}\left[\begin{array}{l}
\left(\lambda_{1}: 1,1,1\right),\left(\lambda_{2}: 1,1,1\right),\left(1+\alpha_{1}+\beta_{1}: 1,2,1\right),\left(1+\alpha_{2}+\beta_{2}: 1,1,2\right): \\
\left(\mu_{1}: 1,1,1\right),\left(\mu_{2}: 1,1,1\right),\left(1+\alpha_{1}+\beta_{1}: 1,1,1\right),\left(1+\alpha_{2}+\beta_{2}: 1,1,1\right):
\end{array}\right. \\
& \left.\begin{array}{lll}
-;\left(\frac{1}{2}\left(1+z_{1}\right): 1\right) & ;\left(\frac{1}{2}\left(1+z_{2}\right): 1\right) & ; \\
- & ;\left(1+\alpha_{1}: 1\right),\left(p_{1}: 1\right) & ;\left(1+\alpha_{2}: 1\right),\left(p_{2}: 1\right)
\end{array} ;,-t,-t\right] .
\end{aligned}
$$

\section{Special cases}

If we put $\lambda=\mu$ in the generating function (5.3), we get

$$
\begin{aligned}
& \sum_{n=0}^{\infty} \frac{1}{n !} F_{n}^{\left(\alpha_{1}, \beta_{1} ; \alpha_{2}, \beta_{2}\right)}\left(p_{1}, z_{1} ; p_{2}, z_{2}\right) t^{n} \\
& =F_{2: 0 ; 2 ; 2}^{2: 0 ; 1 ; 1}\left[\begin{array}{l}
\left(1+\alpha_{1}+\beta_{1}: 1,2,1\right),\left(1+\alpha_{2}+\beta_{2}: 1,1,2\right):-; \\
\left(1+\alpha_{1}+\beta_{1}: 1,1,1\right),\left(1+\alpha_{2}+\beta_{2}: 1,1,1\right):-;
\end{array}\right. \\
& \left.\begin{array}{ll}
\left(\frac{1}{2}\left(1+z_{1}\right): 1\right) & ;\left(\frac{1}{2}\left(1+z_{2}\right): 1\right) \\
\left(1+\alpha_{1}: 1\right),\left(p_{1}: 1\right) & ;\left(1+\alpha_{2}: 1\right),\left(p_{2}: 1\right)
\end{array} ; t,-t,-t\right] .
\end{aligned}
$$

When $\lambda_{1}=1+\alpha_{1}+\beta_{1}$ and $\lambda_{2}=1+\alpha_{2}+\beta_{2}$, then the generating function (5.4) reduces immediately to the form :

$$
\begin{aligned}
& \sum_{n=0}^{\infty} \frac{\left(1+\alpha_{1}+\beta_{1}\right)_{n}\left(1+\alpha_{2}+\beta_{2}\right)_{n}}{n !(\mu)_{n}} F_{n}^{\left(\alpha_{1}, \beta_{1} ; \alpha_{2}, \beta_{2}\right)}\left(p_{1}, z_{1} ; p_{2}, z_{2}\right) t^{n} \\
& =F_{1: 0 ; 2 ; 2}^{2: 0 ; 1 ; 1}\left[\begin{array}{ll}
\left(1+\alpha_{1}+\beta_{1}: 1,2,1\right),\left(1+\alpha_{2}+\beta_{2}: 1,1,2\right) & :-; \\
(\mu: 1,1,1) & :-;
\end{array}\right. \\
& \left.\begin{array}{ll}
\left(\frac{1}{2}\left(1+z_{1}\right): 1\right) & ;\left(\frac{1}{2}\left(1+z_{2}\right): 1\right) \\
\left(1+\alpha_{1}: 1\right),\left(p_{1}: 1\right) & ;\left(1+\alpha_{2}: 1\right),\left(p_{2}: 1\right)
\end{array} ; t,-t,-t\right] .
\end{aligned}
$$

Further, we have the following generating functions:

$$
\begin{aligned}
& \sum_{n=0}^{\infty} \frac{1}{n !} F_{n}^{\left(\alpha_{1}, \beta_{1}-n ; \alpha_{2}, \beta_{2}-n\right)}\left(p_{1}, z_{1} ; p_{2}, z_{2}\right) t^{n} \\
& =e_{2}^{t} F_{2}\left[\begin{array}{c}
1+\alpha_{1}+\beta_{1}, \frac{1}{2}\left(1+z_{1}\right) ; \\
1+\alpha_{1}, p_{1} ;
\end{array}-t\right]{ }_{2} F_{2}\left[\begin{array}{c}
1+\alpha_{2}+\beta_{2}, \frac{1}{2}\left(1+z_{2}\right) ; \\
1+\alpha_{2}, p_{2} ;
\end{array}\right] \text {; } \\
& \sum_{n=0}^{\infty} \frac{\left(-\alpha_{1}\right)_{n}}{n !} F_{n}^{\left(\alpha_{1}-n, \beta_{1} ; \alpha_{2}, \beta_{2}-n\right)}\left(p_{1}, z_{1} ; p_{2}, z_{2}\right) t^{n} \\
& =(1-t)^{\alpha_{1}}{ }_{2} F_{1}\left[\begin{array}{cc}
1+\alpha_{1}+\beta_{1}, \frac{1}{2}\left(1+z_{1}\right) & ; \\
p_{1} & ;
\end{array}\right] \\
& \times{ }_{3} F_{2}\left[\begin{array}{cc}
-\alpha_{1}, 1+\alpha_{2}+\beta_{2}, \frac{1}{2}\left(1+z_{2}\right) & ; \frac{-t}{1-t}
\end{array}\right] ;
\end{aligned}
$$




$$
\begin{aligned}
& \sum_{n=0}^{\infty} \frac{\left(-\alpha_{2}\right)_{n}}{n !} F_{n}^{\left(\alpha_{1}, \beta_{1}-n ; \alpha_{2}-n, \beta_{2}\right)}\left(p_{1}, z_{1} ; p_{2}, z_{2}\right) t^{n} \\
& =(1-t)^{\alpha_{2}} F_{1}\left[\begin{array}{cc}
1+\alpha_{2}+\beta_{2}, \frac{1}{2}\left(1+z_{2}\right) & ; \\
p_{2} & ;
\end{array}\right] \\
& \times{ }_{3} F_{2}\left[\begin{array}{cc}
-\alpha_{2}, 1+\alpha_{1}+\beta_{1}, \frac{1}{2}\left(1+z_{1}\right) & ; \frac{-t}{1-t} \\
1+\alpha_{1}, p_{1} & ;
\end{array} ;\right. \\
& \sum_{n=0}^{\infty} \frac{\left(1-p_{1}\right)_{n}}{n !} F_{n}^{\left(\alpha_{1}, \beta_{1}-n ; \alpha_{2}, \beta_{2}-n\right)}\left(p_{1}-n, z_{1} ; p_{2}, z_{2}\right) t^{n} \\
& =(1-t)^{p_{1}-1}{ }_{2} F_{1}\left[\begin{array}{cl}
1+\alpha_{1}+\beta_{1}, \frac{1}{2}\left(1+z_{1}\right) & ; t \\
1+\alpha_{1} & ;
\end{array}\right] \\
& \times{ }_{3} F_{2}\left[\begin{array}{cc}
1+\alpha_{2}+\beta_{2}, \frac{1}{2}\left(1+z_{2}\right), 1-p_{1} & ; \frac{-t}{1-t} \\
1+\alpha_{2}, p_{2} & ;
\end{array}\right. \text {; } \\
& \sum_{n=0}^{\infty} \frac{\left(1-p_{2}\right)_{n}}{n !} F_{n}^{\left(\alpha_{1}, \beta_{1}-n ; \alpha_{2}, \beta_{2}-n\right)}\left(p_{1}, z_{1} ; p_{2}-n, z_{2}\right) t^{n} \\
& =(1-t)^{p_{2}-1}{ }_{2} F_{1}\left[\begin{array}{cc}
1+\alpha_{2}+\beta_{2}, \frac{1}{2}\left(1+z_{2}\right) & ; \\
1+\alpha_{2} & ;
\end{array}\right] \\
& \times{ }_{3} F_{2}\left[\begin{array}{cc}
1+\alpha_{1}+\beta_{1}, \frac{1}{2}\left(1+z_{1}\right), 1-p_{2} \\
1+\alpha_{1}, p_{1}
\end{array} ; \frac{-t}{1-t}\right] \text {; }
\end{aligned}
$$

$$
\begin{aligned}
& \sum_{n=0}^{\infty} \frac{(\lambda)_{n}}{n !} F_{n}^{\left(\alpha_{1}, \beta_{1}-n ; \alpha_{2}, \beta_{2}-n\right)}\left(p_{1}, z_{1} ; p_{2}, z_{2}\right) t^{n} \\
= & (1-t)^{-\lambda} \\
& \times F_{0: 2 ; 2}^{1: 2 ; 2}\left[\begin{array}{ll}
\lambda: 1+\alpha_{1}+\beta_{1}, \frac{1}{2}\left(1+z_{1}\right) & ; 1+\alpha_{2}+\beta_{2}, \frac{1}{2}\left(1+z_{2}\right) ; \frac{-t}{1-t}, \frac{-t}{1-t} \\
-: 1+\alpha_{1}, p_{1} & ; 1+\alpha_{2}, p_{2}
\end{array}\right.
\end{aligned}
$$

$$
\begin{aligned}
& \sum_{n=0}^{\infty} \frac{\left(-\alpha_{1}\right)_{n}}{n !\left(-\alpha_{1}-\beta_{1}\right)_{n}} F_{n}^{\left(\alpha_{1}-n, \beta_{1}-n ; \alpha_{2}, \beta_{2}-n\right)}\left(p_{1}, z_{1} ; p_{2}, z_{2}\right) t^{n} \\
= & \left.\left.{ }_{1} F_{1}\left[\begin{array}{cc}
\frac{1}{2}\left(1+z_{1}\right) ; \\
p_{1} ;
\end{array}\right]+t\right] F_{1: 0 ; 2}^{1: 0 ; 2}\left[\begin{array}{cc}
-\alpha_{1} & :-; 1+\alpha_{2}+\beta_{2}, \frac{1}{2}\left(1+z_{2}\right) ; \\
-\alpha_{1}-\beta_{1}:-; 1+\alpha_{2}, p_{2} & ;
\end{array}\right], t\right]
\end{aligned}
$$

$$
\begin{aligned}
& \sum_{n=0}^{\infty} \frac{\left(-\alpha_{2}\right)_{n}}{n !\left(-\alpha_{2}-\beta_{2}\right)_{n}} F_{n}^{\left(\alpha_{1}, \beta_{1}-n ; \alpha_{2}-n, \beta_{2}-n\right)}\left(p_{1}, z_{1} ; p_{2}, z_{2}\right) t^{n} \\
= & { }_{1} F_{1}\left[\begin{array}{cc}
\frac{1}{2}\left(1+z_{2}\right) ; \\
p_{2} ;
\end{array} ; t F_{1: 0 ; 2}^{1: 0 ; 2}\left[\begin{array}{cc}
-\alpha_{2} & :-; 1+\alpha_{1}+\beta_{1}, \frac{1}{2}\left(1+z_{1}\right) ; \\
-\alpha_{2}-\beta_{2}:-; 1+\alpha_{1}, p_{1} & ;
\end{array}\right],-t\right]
\end{aligned}
$$


(5.16)

$$
\begin{aligned}
& \sum_{n=0}^{\infty} \frac{\left(1-p_{1}\right)_{n}}{n !\left(-\alpha_{1}-\beta_{1}\right)_{n}} F_{n}^{\left(\alpha_{1}, \beta_{1}-2 n ; \alpha_{2}, \beta_{2}-n\right)}\left(p_{1}-n, z_{1} ; p_{2}, z_{2}\right) t^{n} \\
& ={ }_{1} F_{1}\left[\begin{array}{c}
\frac{1}{2}\left(1+z_{1}\right) ; \\
1+\alpha_{1} ;
\end{array} ; t\right] F_{1: 0 ; 2}^{1: 0 ; 2}\left[\begin{array}{cc}
1-p_{1}:-; 1+\alpha_{2}+\beta_{2}, \frac{1}{2}\left(1+z_{2}\right) ; \\
-\alpha_{1}-\beta_{1}:-; 1+\alpha_{2}, p_{2}
\end{array} ;,-t\right] \text {; } \\
& \sum_{n=0}^{\infty} \frac{\left(1-p_{2}\right)_{n}}{n !\left(-\alpha_{2}-\beta_{2}\right)_{n}} F_{n}^{\left(\alpha_{1}, \beta_{1}-n ; \alpha_{2}, \beta_{2}-2 n\right)}\left(p_{1}, z_{1} ; p_{2}-n, z_{2}\right) t^{n} \\
& ={ }_{1} F_{1}\left[\begin{array}{c}
\frac{1}{2}\left(1+z_{2}\right) ; \\
1+\alpha_{2} ;
\end{array} ; t\right] F_{1: 0 ; 2}^{1: 0 ; 2}\left[\begin{array}{cc}
1-p_{2}:-; 1+\alpha_{1}+\beta_{1}, \frac{1}{2}\left(1+z_{1}\right) ; \\
-\alpha_{2}-\beta_{2}:-; 1+\alpha_{1}, p_{1}
\end{array} ;,-t\right] .
\end{aligned}
$$

Also, we have the following generating functions:

$$
\begin{aligned}
& \sum_{n=0}^{\infty} \frac{(\lambda)_{n}}{n !(\mu)_{n}} F_{n}^{\left(\alpha_{1}, \beta_{1}-n ; \alpha_{2}, \beta_{2}-n\right)}\left(p_{1}, z_{1} ; p_{2}, z_{2}\right) t^{n} \\
= & F^{(3)}\left[\begin{array}{ccc}
\lambda::-;-;-:-; 1+\alpha_{1}+\beta_{1}, \frac{1}{2}\left(1+z_{1}\right) & \left.; 1+\alpha_{2}+\beta_{2}, \frac{1}{2}\left(1+z_{2}\right) ; t,-t,-t\right] ; \\
\mu::-;-;-:-; 1+\alpha_{1}, p_{1} & ; 1+\alpha_{2}, p_{2}
\end{array}\right.
\end{aligned}
$$

$$
\begin{aligned}
& \sum_{n=0}^{\infty} \frac{\left(-\alpha_{1}\right)_{n}\left(-\alpha_{2}\right)_{n}}{n !} F_{n}^{\left(\alpha_{1}-n, \beta_{1} ; \alpha_{2}-n, \beta_{2}\right)}\left(p_{1}, z_{1} ; p_{2}, z_{2}\right) t^{n} \\
= & \left.F^{(3)}\left[\begin{array}{llll}
-::-\alpha_{2} & ;-;-\alpha_{1}:-; 1+\alpha_{1}+\beta_{1}, \frac{1}{2}\left(1+z_{1}\right) & ; 1+\alpha_{2}+\beta_{2}, \frac{1}{2}\left(1+z_{2}\right) ; \\
-::- & ;-;- & :-; p_{1} & ; p_{2}
\end{array}\right], t, t\right] ;
\end{aligned}
$$

$$
\begin{aligned}
& \sum_{n=0}^{\infty} \frac{\left(1-p_{1}\right)_{n}\left(1-p_{2}\right)_{n}}{n !} F_{n}^{\left(\alpha_{1}, \beta_{1}-n ; \alpha_{2}, \beta_{2}-n\right)}\left(p_{1}-n, z_{1} ; p_{2}-n, z_{2}\right) t^{n} \\
= & F^{(3)}\left[\begin{array}{ccc}
-:: 1-p_{2} ;-; 1-p_{1}:-; 1+\alpha_{1}+\beta_{1}, \frac{1}{2}\left(1+z_{1}\right) ; 1+\alpha_{2}+\beta_{2}, \frac{1}{2}\left(1+z_{2}\right) ; \\
-::-\quad ;-;-\quad:-; 1+\alpha_{1} & ; 1+\alpha_{2}
\end{array}\right] ;
\end{aligned}
$$

$$
\begin{aligned}
& \sum_{n=0}^{\infty} \frac{\left(-\alpha_{1}\right)_{n}\left(-\alpha_{2}\right)_{n}}{n !\left(-\alpha_{1}-\beta_{1}\right)_{n}\left(-\alpha_{2}-\beta_{2}\right)_{n}} F_{n}^{\left(\alpha_{1}-n, \beta_{1}-n ; \alpha_{2}-n, \beta_{2}-n\right)}\left(p_{1}, z_{1} ; p_{2}, z_{2}\right) t^{n}
\end{aligned}
$$

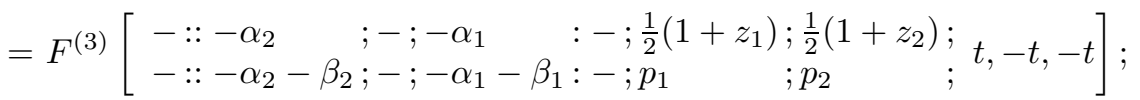

$$
\begin{aligned}
& \sum_{n=0}^{\infty} \frac{\left(1-p_{1}\right)_{n}\left(1-p_{2}\right)_{n}}{n !\left(-\alpha_{1}-\beta_{1}\right)_{n}\left(-\alpha_{2}-\beta_{2}\right)_{n}} F_{n}^{\left(\alpha_{1}, \beta_{1}-2 n ; \alpha_{2}, \beta_{2}-2 n\right)}\left(p_{1}-n, z_{1} ; p_{2}-n, z_{2}\right) t^{n} \\
= & F^{(3)}\left[\begin{array}{ccc}
-:: 1-p_{2} \quad ;-; 1-p_{1} & \left.:-; \frac{1}{2}\left(1+z_{1}\right) ; \frac{1}{2}\left(1+z_{2}\right) ; t,-t,-t\right] . \\
-::-\alpha_{2}-\beta_{2} ;-;-\alpha_{1}-\beta_{1}:-; 1+\alpha_{1} & ; 1+\alpha_{2} \quad ;
\end{array}\right]
\end{aligned}
$$




\section{Generalized Pasternak's polynomials of two variables} by

$\begin{aligned} & (6.1) \\ & F_{n, m_{1}, m_{2}}^{\left(\alpha_{1}, \beta_{1} ; \alpha_{2}, \beta_{2}\right)}\left(z_{1}, z_{2}\right) \\ = & \sum_{r=0}^{n} \sum_{s=0}^{n-r} \frac{(-n)_{r+s}\left(1+\alpha_{1}+\beta_{1}+n\right)_{r}\left(\frac{1}{2}\left(1+z_{1}+m_{1}\right)\right)_{r}\left(1+\alpha_{2}+\beta_{2}+n\right)_{s}\left(\frac{1}{2}\left(1+z_{2}+m_{2}\right)\right)_{s}}{r ! s !\left(1+\alpha_{1}\right)_{r}\left(1+m_{1}\right)_{r}\left(1+\alpha_{2}\right)_{s}\left(1+m_{2}\right)_{s}} .\end{aligned}$

The relation (6.1) can also be expressed in terms of double hypergeometric function as follows:

(6.2)

$$
\begin{aligned}
& F_{n, m_{1}, m_{2}}^{\left(\alpha_{1}, \beta_{1} ; \alpha_{2}, \beta_{2}\right)}\left(z_{1}, z_{2}\right) \\
= & F_{0: 2 ; 2}^{1: 2 ; 2}\left[\begin{array}{lll}
-n: 1+\alpha_{1}+\beta_{1}+n, \frac{1}{2}\left(1+z_{1}+m_{1}\right) & \left.; 1+\alpha_{2}+\beta_{2}+n, \frac{1}{2}\left(1+z_{2}+m_{2}\right) ; 1,1\right] \\
-: 1+\alpha_{1}, 1+m_{1} & ; 1+\alpha_{2}, 1+m_{2}
\end{array}\right]
\end{aligned}
$$

where we have used a special case of the double hypergeometric function defined by (1.4).

The definition (6.1) can also be represented as follows:

$$
\begin{aligned}
& F_{n, m_{1}, m_{2}}^{\left(\alpha_{1}, \beta_{1} ; \alpha_{2}, \beta_{2}\right)}\left(z_{1}, z_{2}\right) \\
= & \sum_{r=0}^{n} \frac{(-n)_{r}\left(1+\alpha_{1}+\beta_{1}+n\right)_{r}\left(\frac{1}{2}\left(1+z_{1}+m_{1}\right)\right)_{r}}{r !\left(1+\alpha_{1}\right)_{r}\left(1+m_{1}\right)_{r}} F_{n-r, m_{2}}^{\left(\alpha_{2}, \beta_{2}+r\right)}\left(z_{2}\right),
\end{aligned}
$$

where $F_{n, m_{2}}^{(\alpha, \beta)}\left(z_{2}\right)$ is the well known generalized Pasternak's polynomial of one variable defined by (1.3).

The relationships between generalized Pasternak's polynomials of two variables and generalized Pasternak's polynomials of single variable are as follows:

$$
F_{n, m_{1}, m_{2}}^{\left(\alpha_{1}, \beta_{1} ; \alpha_{2}, \beta_{2}\right)}\left(z_{1},-1-m_{2}\right)=F_{n, m_{1}}^{\left(\alpha_{1}, \beta_{1}\right)}\left(z_{1}\right)
$$

and

$$
F_{n, m_{1}, m_{2}}^{\left(\alpha_{1}, \beta_{1} ; \alpha_{2}, \beta_{2}\right)}\left(-1-m_{1}, z_{2}\right)=F_{n, m_{2}}^{\left(\alpha_{2}, \beta_{2}\right)}\left(z_{2}\right) .
$$




\section{Generating functions for generalized Pasternak's polynomials of two variables}

The polynomials $F_{n, m_{1}, m_{2}}^{\left(\alpha_{1}, \beta_{1} ; \alpha_{2}, \beta_{2}\right)}\left(z_{1}, z_{2}\right)$ admit the following generating functions:

$(7.1)$

$$
\begin{aligned}
& \sum_{n=0}^{\infty} \frac{(\lambda)_{n}}{n !} F_{n, m_{1}, m_{2}}^{\left(\alpha_{1}, \beta_{1} ; \alpha_{2}, \beta_{2}\right)}\left(z_{1}, z_{2}\right) t^{n} \\
& =F_{2: 0 ; 2 ; 2}^{3: 0 ; 1 ; 1}\left[\begin{array}{ll}
(\lambda: 1,1,1),\left(1+\alpha_{1}+\beta_{1}: 1,2,1\right),\left(1+\alpha_{2}+\beta_{2}: 1,1,2\right):-; \\
\left(1+\alpha_{1}+\beta_{1}: 1,1,1\right),\left(1+\alpha_{2}+\beta_{2}: 1,1,1\right) & :-;
\end{array}\right. \\
& \left.\begin{array}{lll}
\left(\frac{1}{2}\left(1+z_{1}+m_{1}\right): 1\right) & ;\left(\frac{1}{2}\left(1+z_{2}+m_{2}\right): 1\right) & ; \\
\left(1+\alpha_{1}: 1\right),\left(1+m_{1}: 1\right) & ;\left(1+\alpha_{2}: 1\right),\left(1+m_{2}: 1\right)
\end{array} ;,-t,-t\right],
\end{aligned}
$$

where we have used a special case of generalized Lauricella function (see, for example, [5, p. 37]).

(7.2)

$$
\begin{aligned}
& \sum_{n=0}^{\infty} \frac{1}{n !(\mu)_{n}} F_{n, m_{1}, m_{2}}^{\left(\alpha_{1}, \beta_{1} ; \alpha_{2}, \beta_{2}\right)}\left(z_{1}, z_{2}\right) t^{n} \\
& =F_{3: 0 ; 2 ; 2}^{2: 0 ; 1 ; 1}\left[\begin{array}{ll}
\left(1+\alpha_{1}+\beta_{1}: 1,2,1\right),\left(1+\alpha_{2}+\beta_{2}: 1,1,2\right) & :-; \\
(\mu: 1,1,1),\left(1+\alpha_{1}+\beta_{1}: 1,1,1\right),\left(1+\alpha_{2}+\beta_{2}: 1,1,1\right) & :-;
\end{array}\right. \\
& \left.\begin{array}{lll}
\left(\frac{1}{2}\left(1+z_{1}+m_{1}\right): 1\right) & ;\left(\frac{1}{2}\left(1+z_{2}+m_{2}\right): 1\right)
\end{array} ; t,-t,-t\right] ;
\end{aligned}
$$

$$
\begin{aligned}
& \sum_{n=0}^{\infty} \frac{(\lambda)_{n}}{n !(\mu)_{n}} F_{n, m_{1}, m_{2}}^{\left(\alpha_{1}, \beta_{1} ; \alpha_{2}, \beta_{2}\right)}\left(z_{1}, z_{2}\right) t^{n} \\
&=F_{3: 0 ; 2 ; 2}^{3: 0 ; 1 ; 1}\left[\begin{array}{ll}
(\lambda: 1,1,1),\left(1+\alpha_{1}+\beta_{1}: 1,2,1\right),\left(1+\alpha_{2}+\beta_{2}: 1,1,2\right):-; \\
(\mu: 1,1,1),\left(1+\alpha_{1}+\beta_{1}: 1,1,1\right),\left(1+\alpha_{2}+\beta_{2}: 1,1,1\right):-; \\
\\
\left(\frac{1}{2}\left(1+z_{1}+m_{1}\right): 1\right) & ;\left(\frac{1}{2}\left(1+z_{2}+m_{2}\right): 1\right) \\
& \left.\left(1+\alpha_{1}: 1\right),\left(1+m_{1}: 1\right) ;\left(1+\alpha_{2}: 1\right),\left(1+m_{2}: 1\right) ; t,-t,-t\right] ;
\end{array}\right.
\end{aligned}
$$

$$
\begin{aligned}
& \sum_{n=0}^{\infty} \frac{\left(\lambda_{1}\right)_{n}\left(\lambda_{2}\right)_{n}}{n !(\mu)_{n}} F_{n, m_{1}, m_{2}}^{\left(\alpha_{1}, \beta_{1} ; \alpha_{2}, \beta_{2}\right)}\left(z_{1}, z_{2}\right) t^{n} \\
&=F_{3: 0 ; 2 ; 2}^{4: 0 ; 1 ; 1}\left[\begin{array}{c}
\left(\lambda_{1}: 1,1,1\right),\left(\lambda_{2}: 1,1,1\right),\left(1+\alpha_{1}+\beta_{1}: 1,2,1\right),\left(1+\alpha_{2}+\beta_{2}: 1,1,2\right): \\
(\mu: 1,1,1),\left(1+\alpha_{1}+\beta_{1}: 1,1,1\right),\left(1+\alpha_{2}+\beta_{2}: 1,1,1\right)
\end{array}\right. \quad ; \\
&\left.-;\left(\frac{1}{2}\left(1+z_{1}+m_{1}\right): 1\right) \quad ;\left(\frac{1}{2}\left(1+z_{2}+m_{2}\right): 1\right) \quad ; t,-t,-t\right] \\
& \quad ;\left(1+\alpha_{1}: 1\right),\left(1+m_{1}: 1\right) ;\left(1+\alpha_{2}: 1\right),\left(1+m_{2}: 1\right) ;
\end{aligned}
$$


$(7.5)$

$$
\begin{aligned}
& \sum_{n=0}^{\infty} \frac{\left(\lambda_{1}\right)_{n}\left(\lambda_{2}\right)_{n}}{n !\left(\mu_{1}\right)_{n}\left(\mu_{2}\right)_{n}} F_{n, m_{1}, m_{2}}^{\left(\alpha_{1}, \beta_{1} ; \alpha_{2}, \beta_{2}\right)}\left(z_{1}, z_{2}\right) t^{n} \\
& =F_{4: 0 ; 2 ; 2}^{4: 0 ; 1 ; 1}\left[\begin{array}{l}
\left(\lambda_{1}: 1,1,1\right),\left(\lambda_{2}: 1,1,1\right),\left(1+\alpha_{1}+\beta_{1}: 1,2,1\right),\left(1+\alpha_{2}+\beta_{2}: 1,1,2\right): \\
\left(\mu_{1}: 1,1,1\right),\left(\mu_{2}: 1,1,1\right),\left(1+\alpha_{1}+\beta_{1}: 1,1,1\right),\left(1+\alpha_{2}+\beta_{2}: 1,1,1\right):
\end{array}\right. \\
& \left.\begin{array}{l}
-;\left(\frac{1}{2}\left(1+z_{1}+m_{1}\right): 1\right) \quad ;\left(\frac{1}{2}\left(1+z_{2}+m_{2}\right): 1\right) \\
-;\left(1+\alpha_{1}: 1\right),\left(1+m_{1}: 1\right) ;\left(1+\alpha_{2}: 1\right),\left(1+m_{2}: 1\right) ;
\end{array} ; t,-t,-t\right] .
\end{aligned}
$$

\section{Special cases}

If we put $\lambda=\mu$ in the generating function (7.3), we get (7.6)

$$
\begin{aligned}
& \sum_{n=0}^{\infty} \frac{1}{n !} F_{n, m_{1}, m_{2}}^{\left(\alpha_{1}, \beta_{1} ; \alpha_{2}, \beta_{2}\right)}\left(z_{1}, z_{2}\right) t^{n} \\
&=F_{2: 0 ; 2 ; 2}^{2: 0 ; 1 ; 1}\left[\begin{array}{ll}
\left(1+\alpha_{1}+\beta_{1}: 1,2,1\right),\left(1+\alpha_{2}+\beta_{2}: 1,1,2\right):-; \\
\left(1+\alpha_{1}+\beta_{1}: 1,1,1\right),\left(1+\alpha_{2}+\beta_{2}: 1,1,1\right):-; \\
& \left(\frac{1}{2}\left(1+z_{1}+m_{1}\right): 1\right) \quad ;\left(\frac{1}{2}\left(1+z_{2}+m_{2}\right): 1\right) \\
& \left.\left(1+\alpha_{1}: 1\right),\left(1+m_{1}: 1\right) ;\left(1+\alpha_{2}: 1\right),\left(1+m_{2}: 1\right) ; t,-t,-t\right] .
\end{array} .\right.
\end{aligned}
$$

When $\lambda_{1}=1+\alpha_{1}+\beta_{1}$ and $\lambda_{2}=1+\alpha_{2}+\beta_{2}$, then the generating function (7.4) reduces immediately to the form:

$$
\begin{aligned}
& \sum_{n=0}^{\infty} \frac{\left(1+\alpha_{1}+\beta_{1}\right)_{n}\left(1+\alpha_{2}+\beta_{2}\right)_{n}}{n !(\mu)_{n}} F_{n, m_{1}, m_{2}}^{\left(\alpha_{1}, \beta_{1} ; \alpha_{2}, \beta_{2}\right)}\left(z_{1}, z_{2}\right) t^{n} \\
&=F_{1: 0 ; 2 ; 2}^{2: 0 ; 1 ; 1}\left[\begin{array}{rr}
\left(1+\alpha_{1}+\beta_{1}: 1,2,1\right),\left(1+\alpha_{2}+\beta_{2}: 1,1,2\right):-; \\
(\mu: 1,1,1) & :-; \\
\left(\frac{1}{2}\left(1+z_{1}+m_{1}\right): 1\right) & ;\left(\frac{1}{2}\left(1+z_{2}+m_{2}\right): 1\right) \\
\left(1+\alpha_{1}: 1\right),\left(1+m_{1}: 1\right) & \left.;\left(1+\alpha_{2}: 1\right),\left(1+m_{2}: 1\right) ; t,-t,-t\right] .
\end{array} .\right.
\end{aligned}
$$

Further, we have the following generating functions:

$$
\begin{aligned}
& \sum_{n=0}^{\infty} \frac{1}{n !} F_{n, m_{1}, m_{2}}^{\left(\alpha_{1}, \beta_{1}-n ; \alpha_{2}, \beta_{2}-n\right)}\left(z_{1}, z_{2}\right) t^{n} \\
= & e^{t}{ }_{2} F_{2}\left[\begin{array}{cc}
1+\alpha_{1}+\beta_{1}, \frac{1}{2}\left(1+z_{1}+m_{1}\right) & ; \\
1+\alpha_{1}, 1+m_{1} & ;
\end{array}\right] \\
& \times{ }_{2} F_{2}\left[\begin{array}{cc}
1+\alpha_{2}+\beta_{2}, \frac{1}{2}\left(1+z_{2}+m_{2}\right) & ; \\
1+\alpha_{2}, 1+m_{2} & ;
\end{array}\right] ;
\end{aligned}
$$




$$
\begin{aligned}
& \sum_{n=0}^{\infty} \frac{\left(-\alpha_{1}\right)_{n}}{n !} F_{n, m_{1}, m_{2}}^{\left(\alpha_{1}-n, \beta_{1} ; \alpha_{2}, \beta_{2}-n\right)}\left(z_{1}, z_{2}\right) t^{n} \\
= & \left.(1-t)^{\alpha_{1}}{ }_{2} F_{1}\left[\begin{array}{c}
1+\alpha_{1}+\beta_{1}, \frac{1}{2}\left(1+z_{1}+m_{1}\right) \\
1+m_{1}
\end{array}\right) ; t\right] \\
& \times{ }_{3} F_{2}\left[\begin{array}{c}
-\alpha_{1}, 1+\alpha_{2}+\beta_{2}, \frac{1}{2}\left(1+z_{2}+m_{2}\right) \\
1+\alpha_{2}, 1+m_{2}
\end{array} ; \frac{-t}{1-t}\right] ; \\
& \sum_{n=0}^{\infty} \frac{\left(-\alpha_{2}\right)_{n}}{n !} F_{n, m_{1}, m_{2}}^{\left(\alpha_{1}, \beta_{1}-n, \alpha_{2}-n, \beta_{2}\right)}\left(z_{1}, z_{2}\right) t^{n} \\
= & (1-t)^{\alpha_{2}}{ }_{2} F_{1}\left[\begin{array}{c}
1+\alpha_{2}+\beta_{2}, \frac{1}{2}\left(1+z_{2}+m_{2}\right) \\
1+m_{2}
\end{array}\right. \\
& \times{ }_{3} F_{2}\left[\begin{array}{c}
-\alpha_{2}, 1+\alpha_{1}+\beta_{1}, \frac{1}{2}\left(1+z_{1}+m_{1}\right) \\
1+\alpha_{1}, 1+m_{1}
\end{array}\right]
\end{aligned}
$$

(7.11)

$$
\begin{aligned}
& \sum_{n=0}^{\infty} \frac{(\lambda)_{n}}{n !} F_{n, m_{1}, m_{2}}^{\left(\alpha_{1}, \beta_{1}-n ; \alpha_{2}, \beta_{2}-n\right)}\left(z_{1}, z_{2}\right) t^{n} \\
= & (1-t)^{-\lambda} \\
& \times F_{0: 2 ; 2}^{1: 22}\left[\begin{array}{lll}
\lambda: 1+\alpha_{1}+\beta_{1}, \frac{1}{2}\left(1+z_{1}+m_{1}\right) & ; 1+\alpha_{2}+\beta_{2}, \frac{1}{2}\left(1+z_{2}+m_{2}\right) & ; \\
-: 1+\alpha_{1}, 1+m_{1} & ; 1+\alpha_{2}, 1+m_{2}
\end{array}\right]
\end{aligned}
$$

$$
\begin{aligned}
& \sum_{n=0}^{\infty} \frac{1}{n !\left(\frac{1}{2}\left(1-z_{1}-m_{1}\right)\right)_{n}} F_{n, m_{1}, m_{2}}^{\left(\alpha_{1}, \beta_{1}-n ; \alpha_{2}, \beta_{2}-n\right)}\left(z_{1}-n, z_{2}\right) t^{n} \\
= & { }_{1} F_{2}\left[\begin{array}{cc}
1+\alpha_{1}+\beta_{1} \quad ; \\
1+\alpha_{1}, 1+m_{1} ;
\end{array}\right] \\
& \times F_{1: 0 ; 2}^{0: 0 ; 2}\left[\begin{array}{ll}
- & \left.:-; 1+\alpha_{2}+\beta_{2}, \frac{1}{2}\left(1+z_{2}+m_{2}\right) ; t,-t\right] ; \\
\frac{1}{2}\left(1-z_{1}-m_{1}\right):-; 1+\alpha_{2}, 1+m_{2} & ;
\end{array}\right]
\end{aligned}
$$

$$
\begin{aligned}
& \sum_{n=0}^{\infty} \frac{1}{n !\left(\frac{1}{2}\left(1-z_{2}-m_{2}\right)\right)_{n}} F_{n, m_{1}, m_{2}}^{\left(\alpha_{1}, \beta_{1}-n ; \alpha_{2}, \beta_{2}-n\right)}\left(z_{1}, z_{2}-n\right) t^{n} \\
= & { }_{1} F_{2}\left[\begin{array}{cc}
1+\alpha_{2}+\beta_{2} & ; \\
1+\alpha_{2}, 1+m_{2} ; & ;
\end{array}\right] \\
& \times F_{1: 0 ; 2}^{0: 0 ; 2}\left[\begin{array}{ll}
- & \left.:-; 1+\alpha_{1}+\beta_{1}, \frac{1}{2}\left(1+z_{1}+m_{1}\right) ; t,-t\right] ; \\
\frac{1}{2}\left(1-z_{2}-m_{2}\right):-; 1+\alpha_{1}, 1+m_{1}
\end{array}\right.
\end{aligned}
$$




$$
\begin{aligned}
& \sum_{n=0}^{\infty} \frac{\left(-\alpha_{1}\right)_{n}}{n !\left(-\alpha_{1}-\beta_{1}\right)_{n}} F_{n, m_{1}, m_{2}}^{\left(\alpha_{1}-n, \beta_{1}-n ; \alpha_{2}, \beta_{2}-n\right)}\left(z_{1}, z_{2}\right) t^{n} \\
& ={ }_{1} F_{1}\left[\begin{array}{cc}
\frac{1}{2}\left(1+z_{1}+m_{1}\right) & ; \\
1+m_{1} & ;
\end{array}\right] \\
& \times F_{1: 0 ; 2}^{1: 0 ; 2}\left[\begin{array}{lll}
-\alpha_{1} & :-; 1+\alpha_{2}+\beta_{2}, \frac{1}{2}\left(1+z_{2}+m_{2}\right) & ; \\
-\alpha_{1}-\beta_{1} & :-; 1+\alpha_{2}, 1+m_{2} & ;
\end{array}\right] ; \\
& \sum_{n=0}^{\infty} \frac{\left(-\alpha_{2}\right)_{n}}{n !\left(-\alpha_{2}-\beta_{2}\right)_{n}} F_{n, m_{1}, m_{2}}^{\left(\alpha_{1}, \beta_{1}-n ; \alpha_{2}-n, \beta_{2}-n\right)}\left(z_{1}, z_{2}\right) t^{n} \\
& ={ }_{1} F_{1}\left[\begin{array}{c}
\frac{1}{2}\left(1+z_{2}+m_{2}\right) ; \\
1+m_{2}
\end{array} ;-t\right] \\
& \times F_{1: 0 ; 2}^{1: 0 ; 2}\left[\begin{array}{lll}
-\alpha_{2} & :-; 1+\alpha_{1}+\beta_{1}, \frac{1}{2}\left(1+z_{1}+m_{1}\right) & ; \\
-\alpha_{2}-\beta_{2} & :-; 1+\alpha_{1}, 1+m_{1} & ;
\end{array}\right] ; \\
& \sum_{n=0}^{\infty} \frac{1}{n !\left(-\alpha_{1}-\beta_{1}\right)_{n}} F_{n, m_{1}, m_{2}}^{\left(\alpha_{1}, \beta_{1}-2 n ; \alpha_{2}, \beta_{2}-n\right)}\left(z_{1}, z_{2}\right) t^{n} \\
& (7.16)={ }_{1} F_{2}\left[\begin{array}{c}
\frac{1}{2}\left(1+z_{1}+m_{1}\right) ; t \\
1+\alpha_{1}, 1+m_{1} ;
\end{array}\right] \\
& \times F_{1: 0 ; 2}^{0: 0 ; 2}\left[\begin{array}{lll}
- & :-; 1+\alpha_{2}+\beta_{2}, \frac{1}{2}\left(1+z_{2}+m_{2}\right) & ; \\
-\alpha_{1}-\beta_{1}:-; 1+\alpha_{2}, 1+m_{2} & ;
\end{array},-t\right] ; \\
& \sum_{n=0}^{\infty} \frac{1}{n !\left(-\alpha_{2}-\beta_{2}\right)_{n}} F_{n, m_{1}, m_{2}}^{\left(\alpha_{1}, \beta_{1}-n ; \alpha_{2}, \beta_{2}-2 n\right)}\left(z_{1}, z_{2}\right) t^{n} \\
& \times F_{1: 0 ; 2}^{0: 0 ; 2}\left[\begin{array}{lll}
- & :-; 1+\alpha_{1}+\beta_{1}, \frac{1}{2}\left(1+z_{1}+m_{1}\right) ; t,-t \\
-\alpha_{2}-\beta_{2}:-; 1+\alpha_{1}, 1+m_{1} & ;
\end{array}\right] .
\end{aligned}
$$$$
(7.17)={ }_{1} F_{2}\left[\begin{array}{l}
\frac{1}{2}\left(1+z_{2}+m_{2}\right) ; \\
1+\alpha_{2}, 1+m_{2} ; t
\end{array}\right]
$$

Proof of (7.8).

$$
\begin{aligned}
& \sum_{n=0}^{\infty} \frac{1}{n !} F_{n, m_{1}, m_{2}}^{\left(\alpha_{1}, \beta_{1}-n ; \alpha_{2}, \beta_{2}-n\right)}\left(z_{1}, z_{2}\right) t^{n} \\
& =\sum_{n=0}^{\infty} \frac{1}{n !} \sum_{r=0}^{n} \sum_{s=0}^{n-r} \frac{(-n)_{r+s}\left(1+\alpha_{1}+\beta_{1}\right)_{r}\left(1+\alpha_{2}+\beta_{2}\right)_{s}\left(\frac{1}{2}\left(1+z_{1}+m_{1}\right)\right)_{r}\left(\frac{1}{2}\left(1+z_{2}+m_{2}\right)\right)_{s}}{r ! s !\left(1+\alpha_{1}\right)_{r}\left(1+\alpha_{2}\right)_{s}\left(1+m_{1}\right)_{r}\left(1+m_{2}\right)_{s}} t^{n} \\
& =\sum_{n=0}^{\infty} \sum_{r=0}^{\infty} \sum_{s=0}^{\infty} \frac{\left(1+\alpha_{1}+\beta_{1}\right)_{r}\left(1+\alpha_{2}+\beta_{2}\right)_{s}\left(\frac{1}{2}\left(1+z_{1}+m_{1}\right)\right)_{r}\left(\frac{1}{2}\left(1+z_{2}+m_{2}\right)\right)_{s}}{n ! r ! s !\left(1+\alpha_{1}\right)_{r}\left(1+\alpha_{2}\right)_{s}\left(1+m_{1}\right)_{r}\left(1+m_{2}\right)_{s}} t^{n}(-t)^{r}(-t)^{s} \\
& =e^{t}{ }_{2} F_{2}\left[\begin{array}{c}
1+\alpha_{1}+\beta_{1}, \frac{1}{2}\left(1+z_{1}+m_{1}\right) ; \\
1+\alpha_{1}, 1+m_{1} ;
\end{array}-t\right]{ }_{2} F_{2}\left[\begin{array}{c}
1+\alpha_{2}+\beta_{2}, \frac{1}{2}\left(1+z_{2}+m_{2}\right) ; \\
1+\alpha_{2}, 1+m_{2} ;
\end{array}-t\right] \text {. }
\end{aligned}
$$

Where for ${ }_{2} F_{2}$ one is referred to [4]. This completes the proof of (7.8). 
The other results (7.9) to (7.17) will be established by using the similar argument as in (7.8).

Further,we have the following generating functions:

$$
\sum_{n=0}^{\infty} \frac{(\lambda)_{n}}{n !(\mu)_{n}} F_{n, m_{1}, m_{2}}^{\left(\alpha_{1}, \beta_{1}-n ; \alpha_{2}, \beta_{2}-n\right)}\left(z_{1}, z_{2}\right) t^{n}
$$

$$
\begin{aligned}
& =F^{(3)}\left[\begin{array}{llllll}
\lambda & ::- & ;- & ;- & :- & ; 1+\alpha_{1}+\beta_{1}, \frac{1}{2}\left(1+z_{1}+m_{1}\right) \\
\mu & ::- & ;- & ;- & :- & ; 1+\alpha_{1}, 1+m_{1}
\end{array}\right. \\
& \left.\begin{array}{ll}
1+\alpha_{2}+\beta_{2}, \frac{1}{2}\left(1+z_{2}+m_{2}\right) & ; \\
1+\alpha_{2}, 1+m_{2} & ;
\end{array},-t,-t\right] ;
\end{aligned}
$$

$$
\sum_{n=0}^{\infty} \frac{\left(-\alpha_{1}\right)_{n}\left(-\alpha_{2}\right)_{n}}{n !} F_{n, m_{1}, m_{2}}^{\left(\alpha_{1}-n, \beta_{1} ; \alpha_{2}-n, \beta_{2}\right)}\left(z_{1}, z_{2}\right) t^{n}
$$

$$
\begin{gathered}
=F^{(3)}\left[\begin{array}{lll}
-::-\alpha_{2} & ;-;-\alpha_{1}:-; 1+\alpha_{1}+\beta_{1}, \frac{1}{2}\left(1+z_{1}+m_{1}\right) \\
-::-\quad ;-;-\quad:-; 1+m_{1} \\
1+\alpha_{2}+\beta_{2}, \frac{1}{2}\left(1+z_{2}+m_{2}\right) ; \\
1+m_{2}
\end{array} ; \text {; t, t, }\right] ;
\end{gathered}
$$

$$
\begin{aligned}
& \sum_{n=0}^{\infty} \frac{1}{n !\left(\frac{1}{2}\left(1-z_{1}-m_{1}\right)\right)_{n}\left(\frac{1}{2}\left(1-z_{2}-m_{2}\right)\right)_{n}} \\
& F_{n, m_{1}, m_{2}}^{\left(\alpha_{1}, \beta_{1}-n ; \alpha_{2}, \beta_{2}-n\right)}\left(z_{1}-n, z_{2}-n\right) t^{n} \\
& =F^{(3)}\left[\begin{array}{lll}
-::- & ;-;- & :-; 1+\alpha_{1}+\beta_{1} \\
-:: \frac{1}{2}\left(1-z_{2}-m_{2}\right) & ;-; \frac{1}{2}\left(1-z_{1}-m_{1}\right) & :-; 1+\alpha_{1}, 1+m_{1}
\end{array} ;\right. \\
& \left.\begin{array}{l}
1+\alpha_{2}+\beta_{2} \\
1+\alpha_{2}, 1+m_{2}
\end{array} ; t, t, t\right] \\
& \sum_{n=0}^{\infty} \frac{\left(-\alpha_{1}\right)_{n}\left(-\alpha_{2}\right)_{n}}{n !\left(-\alpha_{1}-\beta_{1}\right)_{n}\left(-\alpha_{2}-\beta_{2}\right)_{n}} F_{n, m_{1}, m_{2}}^{\left(\alpha_{1}-n, \beta_{1}-n ; \alpha_{2}-n, \beta_{2}-n\right)}\left(z_{1}, z_{2}\right) t^{n} \\
& (7.21)=F^{(3)}\left[\begin{array}{lll}
-::-\alpha_{2} & ;-;-\alpha_{1} & :-; \frac{1}{2}\left(1+z_{1}+m_{1}\right) \\
-::-\alpha_{2}-\beta_{2} & ;-;-\alpha_{1}-\beta_{1}:-; 1+m_{1}
\end{array} ;\right.
\end{aligned}
$$

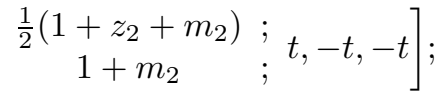

$$
\begin{aligned}
& \sum_{n=0}^{\infty} \frac{1}{n !\left(-\alpha_{1}-\beta_{1}\right)_{n}\left(-\alpha_{2}-\beta_{2}\right)_{n}} F_{n, m_{1}, m_{2}}^{\left(\alpha_{1}, \beta_{1}-2 n ; \alpha_{2}, \beta_{2}-2 n\right)}\left(z_{1}, z_{2}\right) t^{n} \\
& =F^{(3)}\left[\begin{array}{lll}
-::- & ;-;- & :-; \frac{1}{2}\left(1+z_{1}+m_{1}\right) \\
-::-\alpha_{2}-\beta_{2} & ;-;-\alpha_{1}-\beta_{1}:-; 1+\alpha_{1}, 1+m_{1}
\end{array} ;\right.
\end{aligned}
$$

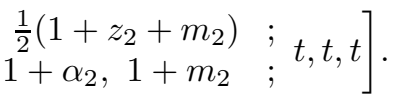


Proof of (7.18).

$$
\begin{aligned}
& \sum_{n=0}^{\infty} \frac{(\lambda)_{n}}{n !(\mu)_{n}} F_{n, m_{1}, m_{2}}^{\left(\alpha_{1}, \beta_{1}-n ; \alpha_{2}, \beta_{2}-n\right)}\left(z_{1}, z_{2}\right) t^{n} \\
& =\sum_{n=0}^{\infty} \frac{(\lambda)_{n}}{n !(\mu)_{n}} \sum_{r=0}^{n} \sum_{s=0}^{n-r} \frac{(-n)_{r+s}\left(1+\alpha_{1}+\beta_{1}\right)_{r}\left(1+\alpha_{2}+\beta_{2}\right)_{s}\left(\frac{1}{2}\left(1+z_{1}+m_{1}\right)\right)_{r}\left(\frac{1}{2}\left(1+z_{2}+m_{2}\right)\right)_{s}}{r ! s !\left(1+\alpha_{1}\right)_{r}\left(1+\alpha_{2}\right)_{s}\left(1+m_{1}\right)_{r}\left(1+m_{2}\right)_{s}} t^{n} \\
& =\sum_{n=0}^{\infty} \sum_{r=0}^{n} \sum_{s=0}^{n-r} \frac{(-1)^{r+s}(\lambda)_{n}\left(1+\alpha_{1}+\beta_{1}\right)_{r}\left(1+\alpha_{2}+\beta_{2}\right)_{s}\left(\frac{1}{2}\left(1+z_{1}+m_{1}\right)\right)_{r}\left(\frac{1}{2}\left(1+z_{2}+m_{2}\right)\right)_{s}}{r ! s !(n-r-s) !(\mu)_{n}\left(1+\alpha_{1}\right)_{r}\left(1+\alpha_{2}\right)_{s}\left(1+m_{1}\right)_{r}\left(1+m_{2}\right)_{s}} t^{n} \\
& =\sum_{n=0}^{\infty} \sum_{r=0}^{\infty} \sum_{s=0}^{\infty} \frac{(\lambda)_{n+r+s}\left(1+\alpha_{1}+\beta_{1}\right)_{r}\left(\frac{1}{2}\left(1+z_{1}+m_{1}\right)\right)_{r}\left(1+\alpha_{2}+\beta_{2}\right)_{s}\left(\frac{1}{2}\left(1+z_{2}+m_{2}\right)\right)_{s}}{n ! r ! s !(\mu)_{n+r+s}\left(1+\alpha_{1}\right)_{r}\left(1+m_{1}\right)_{r}\left(1+\alpha_{2}\right)_{s}\left(1+m_{2}\right)_{s}} \\
& \times t^{n}(-t)^{r}(-t)^{s} \\
& =F^{(3)}\left[\begin{array}{l}
\lambda::-;-;-:-; 1+\alpha_{1}+\beta_{1}, \frac{1}{2}\left(1+z_{1}+m_{1}\right) \\
\mu::-;-;-:-; 1+\alpha_{1}, 1+m_{1}
\end{array} ;\right. \\
& \left.\begin{array}{l}
1+\alpha_{2}+\beta_{2}, \frac{1}{2}\left(1+z_{2}+m_{2}\right) ; \\
1+\alpha_{2}, 1+m_{2}
\end{array} ;,-t,-t\right],
\end{aligned}
$$

where $F^{(3)}[x, y, z]$ denotes a general triple hypergeometric series $[6$, p. 69 , Eq. (39)]. This completes the proof of (7.18).

A similar argument as in getting (7.18) will establish the other results (7.19) to $(7.22)$.

\section{References}

[1] M. A. Khan, A. H. Khan, and S. M. Abbas, On some generating functions of Jacobi polynomials of two variables, submitted for publication.

$[2]$ - On some generating functions of generalized Rice polynomials of two variables, submitted for publication.

[3] M. A. Khan and A. K. Shukla, On some generalized Sister Celine's polynomials, Czechoslovak Math. J. 49(124) (1999), no. 3, 527-545.

[4] E. D. Rainville, Special Functions, Macmillan company, New York, 1960; Reprinted by Chelsea Publishing Company, Bronx, New York, 1971.

[5] H. M. Srivastava and P. W. Karlsson, Multiple Gaussian Hypergeometric Series, Halsted Press (Ellis Horwood Limited, Chichester), John Wiley and Sons, New York, Chichester, Brisbane and Toronto, 1985.

[6] H. M. Srivastava and H. L. Manocha, A Treatise on Generating Functions, Halsted Press (Ellis Horwood Limited, Chichester), John Wiley and Sons, New York, Chichester, Brisbane and Toronto, 1984. 
Sayed Mohammad Abbas

Department of Applied Mathematics

Faculty of Engineering And Technology

Aligarh Muslim University

Aligarh-202002, U. P., India

E-mail address: smabbas.alig@gmail.com

Mumtaz Ahmad Khan

Department of Applied Mathematics

FACUlty of Engineering And Technology

Aligarh Muslim University

Aligarh-202002, U. P., INDIA

E-mail address: mumtaz_ahmad_khan_2008@yahoo.com

ABDUl HAKIM KHAN

Department of Applied Mathematics

Faculty of Engineering And Technology

Aligarh Muslim University

Aligarh-202002, U. P., India

E-mail address: ahkhan.amu@gmail.com 